\title{
Synthesis, characterisation and catalytic properties of vanadium substituted mesoporous aluminophosphates
}

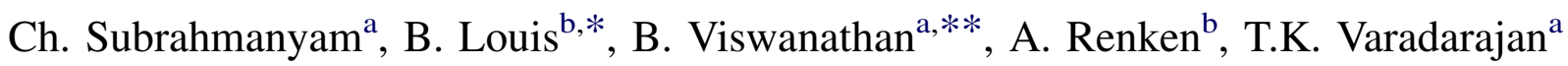 \\ ${ }^{a}$ Department of Chemistry, Indian Institute of Technology Madras, Chennai 600036, India \\ ${ }^{\mathrm{b}}$ Institute of Chemical Engineering, Swiss Federal Institute of Technology, CH-1015 Lausanne, Switzerland
}

Received 20 October 2004; received in revised form 30 November 2004; accepted 1 December 2004

Available online 8 January 2005

\begin{abstract}
Vanadium substituted mesoporous AlPO have been synthesised using structure-directing surfactant under hydrothermal synthesis conditions. The presence of V in the framework of AlPO has been established by ESR, XPS, UV-vis DRS and ICP-AES measurements. This system is capable of promoting the oxidation of toluene to aldehyde and benzoic acid when the oxidising agent is $70 \%$ tert-butyl hydroperoxide while with $30 \% \mathrm{H}_{2} \mathrm{O}_{2}$ cresols are formed. The activity of V-AlPO has been compared with those obtained with other similar porous materials like V-MCM 48, V-MCM-41, V-Al-Beta and VS-1.
\end{abstract}

(C) 2004 Elsevier B.V. All rights reserved.

Keywords: V-AlPO; V-MCM-48; Toluene oxidation; Mesoporous materials; tert-Butyl hydroperoxide (TBHP)

\section{Introduction}

A significant break through in zeolite science occurred in 1982, when Wilson et al. [1] first reported the synthesis of new class of microporous crystalline aluminophosphate materials $\left(\mathrm{AlPO}_{4}\right)$. Later Flanigen et al. [2] reported the successful incorporation of 13 elements into the framework of $\mathrm{AlPO}_{4}-5$. Though these materials are active for various shape selective oxidation reactions, their potential is restricted because of the number and type of heteroelements that can be incorporated into the framework is limited. The pore sizes of the resulting molecular sieves are also another limiting factor.

Synthesis of molecular sieves by structure-directing agents, with an ordering in aggregates of surfactant molecules [3], opens a path to obtain mesoporous molecular sieves with controlled pore sizes and with various other

\footnotetext{
* Corresponding author. Present address: Laboratoire des Matériaux, Surfaces et Procédés pour la Catalyse, LMSPC, 25 rue Becquerel 67087 Strasbourg Cedex 2, France. Tel.: +33 390242 675; fax: +33 390242674 .

E-mail addresses: blouis@chimie.u-strasbg.fr (B. Louis), bviswanathan@hotmail.com (B. Viswanathan).

** Co-corresponding author.
}

elements. Various transition metal ions have been incorporated and the resulting systems are potential catalysts. However, much attention has not been paid to the synthesis of mesoporous aluminophosphates. Efforts to make hexagonal mesoporous aluminophosphates include a first brief report by Kimura et al. [4]. Later the same group reported the formation of stable mesoporous AlPOs using surfactants with long alkyl chains [5,6]. Feng et al. reported the synthesis of mesoporous AlPOs at room temperature through fluoride route [7]. Hexagonal, cubic and lamellar aluminoborates were also prepared [8]. The mechanism involved in the formation of silica based M41S series for the incorporation of silica in AlPO framework was investigated [9]. Attempts have also been made to incorporate transition elements into the framework of mesoporous AlPO [10-18]. Recently, Ti-AlPO has been synthesised [12] and demonstrated as potential catalyst for oxidation of various organic substrates with $30 \% \mathrm{H}_{2} \mathrm{O}_{2}$.

The present communication deals with the formation of $\mathrm{V}$ analogues of mesoporous aluminophosphates via surfactant based hydrothermal synthesis. Their catalytic activity was tested for the partial oxidation of toluene with TBHP and $\mathrm{H}_{2} \mathrm{O}_{2}$ as oxidants and compared with that of V-MCM-48. 


\section{Experimental}

\subsection{Synthesis}

Mesoporous vanadoaluminophosphates were prepared in an optimised procedure using CTAB as surfactant and with the following gel composition. $0.95 \mathrm{Al}_{2} \mathrm{O}_{3}: 1 \mathrm{P}_{2} \mathrm{O}_{5}: 0.05 \mathrm{~V}_{2} \mathrm{O}_{5}$ : 0.4-0.5 CTAB:300 $\mathrm{H}_{2} \mathrm{O}$. Various sources of aluminium have been tried; e.g. nitrate and isopropoxide for instance, and aluminium hydroxide has been chosen as the source. The $\mathrm{pH}$ of the gel was maintained at 9.5 with tetramethyl ammonium hydroxide, as the use of other sources like $\mathrm{NaOH}$ and $\mathrm{NH}_{4} \mathrm{OH}$ led only to amorphous materials. The resulting gel was aged at room temperature for $3 \mathrm{~h}$ followed by autoclaving at $423 \mathrm{~K}$ for $24 \mathrm{~h}$. The solid was filtered, washed several times with deionised water and calcined at $773 \mathrm{~K}$ for $6 \mathrm{~h}$ to remove the organic template.

V-MCM-48 materials were synthesised under hydrothermal conditions at $423 \mathrm{~K}$ in a static stainless-steel autoclave according to the procedure described earlier [1416]. Briefly, to an aqueous solution of cetyltrimethylammonium bromide (CTAB, S.D. Fine Chem.), tetraethylorthosilicate (TEOS, 98\%, Merck-Schuchardt) was added along with required amount of vanadium acetonylacetate (Fluka). The $\mathrm{pH}$ of the gel was adjusted to 10.0-10.5. The typical molar gel composition was $\mathrm{SiO}_{2}: \mathrm{V}_{x} \mathrm{O}_{y}$ :CTAB: $\mathrm{Na}_{2} \mathrm{O}$ :E$\mathrm{tOH}: \mathrm{H}_{2} \mathrm{O}=2.0: 0.015: 0.24: 0.5: 5.0: 195$. The gel was stirred for $3 \mathrm{~h}$ at room temperature and autoclaved at $423 \mathrm{~K}$ for $10 \mathrm{~h}$. The solid product was filtered, washed several times with deionised water, dried in air at $373 \mathrm{~K}$ over night, calcined for $1 \mathrm{~h}$ in $\mathrm{N}_{2}$ atmosphere followed by heating in air at $823 \mathrm{~K}$ for $10 \mathrm{~h}$. Pure silica materials were also prepared by the same procedure. In general, extra-framework cations will mainly contribute to homogeneous catalysis. In order to remove the extra-framework vanadium ions present in the mesoporous catalysts (V-AlPO and V-MCM-48), the calcined materials were washed with ammonium acetate solution ( $1 \mathrm{M}$ ) for $12 \mathrm{~h}$, filtered, dried at $373 \mathrm{~K}$ and calcined at $773 \mathrm{~K}$ for $6 \mathrm{~h}$ in air. Detailed characterisation of V-MCM48 was reported earlier [14-16].

\subsection{Characterisation}

Various techniques have been used for the characterisation of the materials synthesised. The low angle X-ray diffraction pattern of the sample was recorded on a Siemens D $500(\theta / 2 \theta)$ using monochromatized $\mathrm{Cu} \mathrm{K} \alpha$ radiation $(\lambda=1.5406 \AA)$ with a scan speed of $1^{\circ} / \mathrm{min}$ over the range $2^{\circ}<2 \theta<9^{\circ}$. ICP-AES was done with Labtam Plasma Lab 8440. Thermal analysis of the samples was made with thermal analyser (Perkin-Elmer model TGA 7) at a heating rate of $20{ }^{\circ} \mathrm{C} / \mathrm{min}$. Diffuse reflectance UV-vis spectra were recorded in Nujol mode on a Cary 5E UV-vis-NIR spectrophotometer. ESR spectra were recorded with Varian E-112 spectrometer at liquid nitrogen room temperature $(77 \mathrm{~K}) . \mathrm{N}_{2}$ adsorption-desorption at $77 \mathrm{~K}$ measurements were made using a Carlo Erba instruments,
Sorptomatic 1990. The sample was out gassed at $473 \mathrm{~K}$ for $12 \mathrm{~h}$. X-ray photoelectron spectroscopic measurements (XPS) were performed on a PHI-550 ESCA-System (Perkin-Elmer $\mathrm{GmbH}$ ). The $\mathrm{V}_{2 \mathrm{p}}$ region between 510 and $520 \mathrm{eV}$ was studied in detail.

\subsection{Catalytic activity}

Oxidation of toluene was carried out at $333 \mathrm{~K}$ in a threenecked round bottomed flask under reflux conditions using $70 \%$ TBHP as oxidant. Acetone, acetonitrile and methanol were used as solvents. The temperature was maintained by a thermostated oil bath. After each experiment, XRD was recorded to examine the morphological changes. The reaction products were identified by GC-MS (HewlettPackard, HPG 1800A GCD system, HP-5) and analysed by gas chromatography (Shimadzu GC-14A, 30m HP-5. FID).

\section{Results and discussion}

\subsection{XRD measurements}

The XRD pattern of V-AlPO materials is given in Fig. 1. The pattern has been indexed on the basis of a lamellar lattice characteristic of MCM-50 type structure. The assynthesised material shows a maximum intense peak corresponding to $\left(\begin{array}{lll}1 & 0 & 0\end{array}\right)$ reflection followed by broad peaks corresponding to $(200)$ and $(300)$ reflections that can be indexed to a lamellar lattice. An organic base, tetramethylammonium hydroxide (TMAOH), was used to maintain the $\mathrm{pH}$ of the gel. However, with $\mathrm{NaOH}$ and $\mathrm{NH}_{4} \mathrm{OH}$ only amorphous materials were formed. The function of organic ammonium cation from TMAOH is probably to modify the strength of the electrostatic interactions between the aluminophosphate species and the cationic surfactant micelle assembly to form the $\mathrm{S}^{+} \mathrm{I}^{-} / \mathrm{TMA}^{+}$ion pair. If either $\mathrm{NaOH}$ or $\mathrm{NH}_{4} \mathrm{OH}$ is used, the smaller cations $\mathrm{Na}^{+}, \mathrm{NH}_{4}^{+}$ compete with the aluminophosphate species and thus restrict the interaction with the positively charged cationic

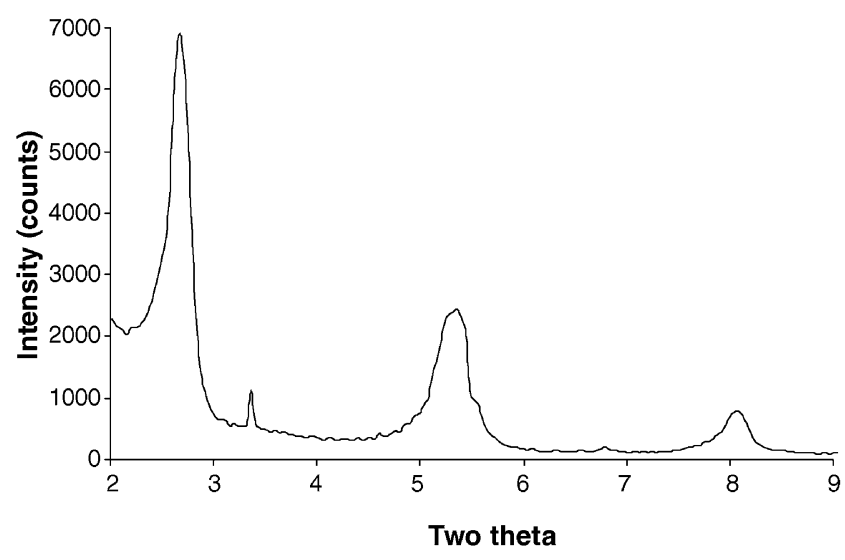

Fig. 1. XRD pattern of V-AlPO. 
surfactant [10]. As stated earlier, aluminium hydroxide may form a less polymerised aluminophosphate with many hydroxyl groups and favour the assembly of the mesostructure compared to other aluminium sources.

\subsection{Thermal analysis}

The thermogram for AlPO samples in the temperature range $323-1073 \mathrm{~K}$, shows mainly two weight loss regions and total weight loss corresponds to $40 \%$. This weight loss includes a first region at 353-393 K corresponding to the loss of physisorbed water. The second and main one in the region $493-533 \mathrm{~K}$ corresponds to the removal of the template. It was observed that AlPO samples are stable up to $1073 \mathrm{~K}$ unlike the stability reported up to $973 \mathrm{~K}$ [10].

\section{3. $\mathrm{N}_{2}$ adsorption}

The $\mathrm{N}_{2}$ adsorption-desorption isotherms for V-MCM-48 and V-AlPO materials are given in Fig. 2. MCM-48 materials showed a clear type IV adsorption-desorption isotherms with a hysteresis loop. The isotherms have an inflection around $P / P_{0}=0.2-0.3$ indicative of mesoporous nature. The surface area is $\sim 845 \mathrm{~m}^{2} / \mathrm{g}$ and average pore size is $28 \AA$. However, in the case of V-AlPO materials, $\mathrm{N}_{2}$ adsorption isotherms is not well resolved like MCM-48, but also showed a hysteresis loop characteristic of mesoporous

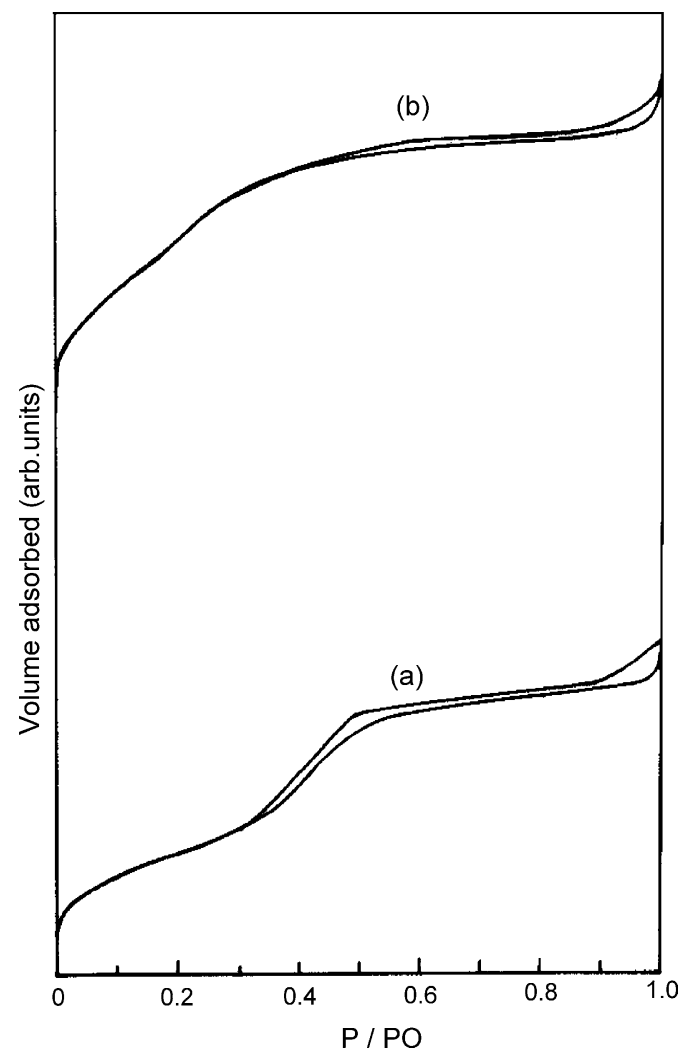

Fig. 2. $\mathrm{N}_{2}$ adsorption-desorption isotherms of (a) V-MCM-48 and (b) VAlPO.

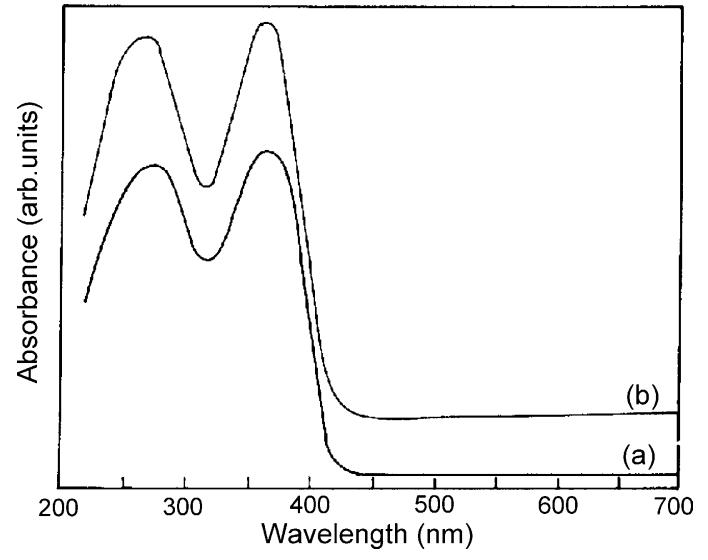

Fig. 3. UV-vis (Nujol) spectra of V-AlPO (a) uncalcined and (b) calcined.

materials located at $P / P_{0}=0.2$. From the shape of the isotherms, it can be concluded that materials are not well ordered like MCM-48. Mesoporous V-AlPO possesses a BET surface area around $650 \mathrm{~m}^{2} / \mathrm{g}$ with a smaller pore size distributed around $20 \AA$.

\section{4. $U V$-vis DRS}

The UV-vis Nujol spectrum of the as-synthesised AlPO sample shows two bands in the region $250-390 \mathrm{~nm}$. The first band in the region $250-300 \mathrm{~nm}$ is characteristic of $\mathrm{V}^{4+}$ charge transfer band of $\mathrm{VO}^{2+}$ species. Moreover, the band in the region 330-390 nm (Fig. 3) is due to charge transfer band resulting from $\mathrm{V}^{5+}$ in $T_{\mathrm{d}}$ environment [19].

\subsection{ESR spectroscopy}

The ESR spectra of as-synthesised and calcined AlPO samples are given in Fig. 4. It exhibits an axially

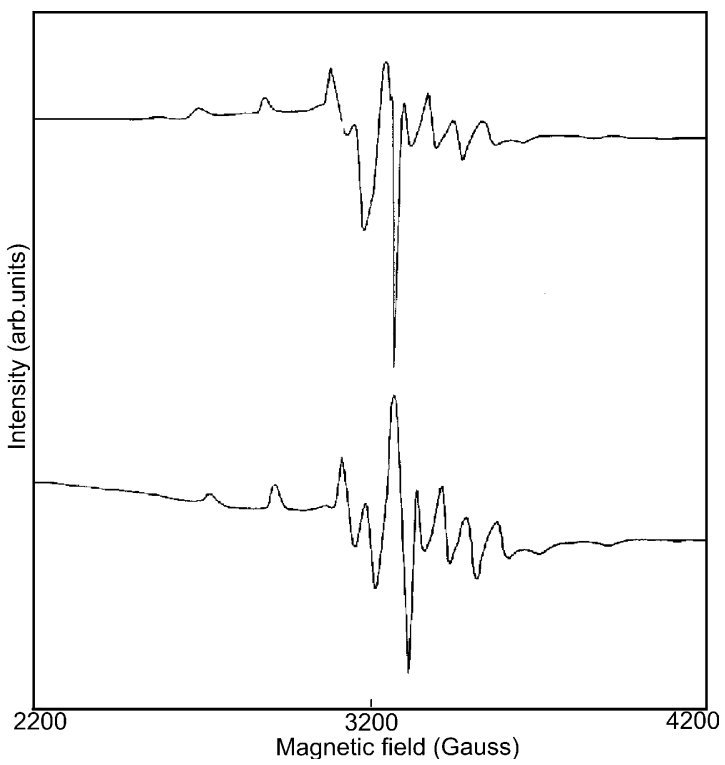

Fig. 4. ESR spectra of V-AlPO (a) uncalcined and (b) calcined. 


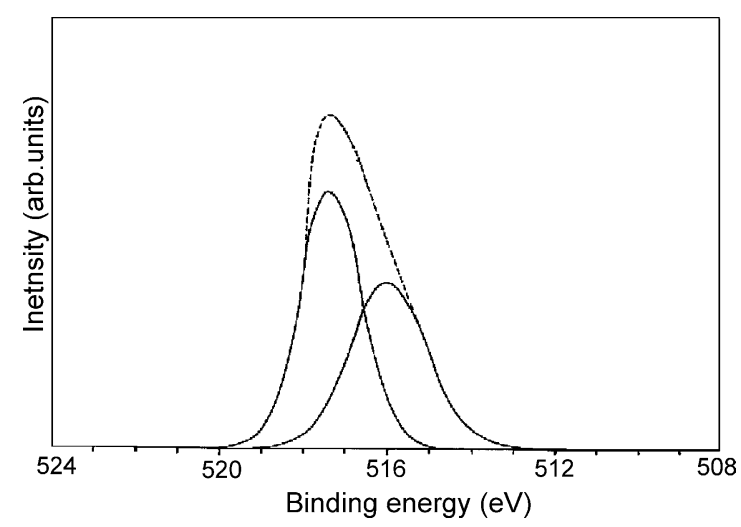

Fig. 5. XPS spectrum of $\mathrm{V}_{2 p}$ region of $\mathrm{V}$-AlPO.

symmetrical signal of tetravalent vanadium, which originated from the $d^{1}$ electron interaction with nuclear spin $\left(I_{\mathrm{n}}=7 / 2\right)$ of ${ }^{51} \mathrm{~V}$. As-synthesised sample exhibits ESR signals with a broad background. The EPR parameters $\left(g_{\|}=1.948, g_{\perp}=1.99, A_{\|}==191 \mathrm{G}, A_{\perp}=70 \mathrm{G}\right)$ indicate that $\mathrm{V}^{4+}$ is present in a distorted $\mathrm{O}_{\mathrm{h}}$ environment [19]. Upon calcination, the signal intensity decreased and sharpness of the signal was increased. This is due to partial conversion of $\mathrm{V}^{4+}$ to $\mathrm{V}^{5+}$ during calcination process.

\subsection{XPS measurements}

The XPS spectra of calcined V-AlPO in $\mathrm{V}_{2 \mathrm{p}}$ region (515$518 \mathrm{eV}$ ) shown in Fig. 5 indicates two peaks: one corresponding to $\mathrm{V}^{4+}$ at $516 \mathrm{eV}$ and other one corresponding to $\mathrm{V}^{5+}$ at $517.4 \mathrm{eV}$. This confirms our contention that during calcination partial oxidation of vanadium takes place. XPS in combination with UV-vis and ESR data confirm the presence both +4 and +5 oxidation states of vanadium.

\subsection{Catalytic activity}

The presence of vanadium in the aluminophosphate framework, or supported between two sheets of the lamellar phase, has been ascertained by various characterisation techniques. ICP-AES analysis of calcined V-AlPO shows 0.5 wt. $\%$ of vanadium. The oxidation of toluene with $70 \%$ TBHP as oxidising agent was carried out with V-AlPO and V-MCM 48 at $333 \mathrm{~K}$ in a variety of solvents and the results are summarised in Tables 1 and 2, respectively. The oxidation of toluene produced benzaldehyde, benzoic acid and benzyl alcohol with high selectivity towards the aldehyde. Interestingly, under the reaction conditions employed for the oxidation of toluene with TBHP, it was observed that both V-AlPO as well as V-MCM-48 have not produced any detectable amount of cresols indicating that these catalysts are promising side-chain oxidation catalysts. When the reaction was performed in the absence of solvent, conversion and selectivity remain low. It was observed that acetone is a better solvent for oxidation of toluene with TBHP compared to other solvents like acetonitrile and methanol. An increase in solvent polarity seems to lead to higher amounts of benzoic acid. The use of protic solvent favours the conversion of the acid formed, through esterification, to form methyl benzoate.

The stability of vanadium containing mesoporous aluminophosphate materials for liquid phase oxidation reactions with TBHP suggests that the aluminophosphate framework $\left(\mathrm{Al}^{3+}, r=0.053 \mathrm{~nm}\right)$ can accommodate vanadium $\left(\mathrm{V}^{4+/ 5+}, r=0.059 / 0.046 \mathrm{~nm}\right)$ centres in a better way than silicate framework $\left(\mathrm{Si}^{4+}, r=0.040 \mathrm{~nm}\right)$ of MCM-48. In order to examine the catalytic activity due to leached metal ions, in a separate experiment, the reaction mixture was filtered after $2 \mathrm{~h}$ under hot conditions and the experiment

Table 1

Catalytic activity of V-AlPO for toluene oxidation with $70 \%$ TBHP

\begin{tabular}{|c|c|c|c|c|c|}
\hline \multirow[t]{2}{*}{ Solvent } & \multirow[t]{2}{*}{ Conversion $(\%)$} & \multicolumn{4}{|c|}{ Product selectivity (\%) } \\
\hline & & Benzaldehyde & Benzoic acid & Benzyl alcohol & Others \\
\hline None & 8.5 & 78.5 & 16.5 & 2.0 & 3.0 \\
\hline Acetone & 27.4 & 76.5 & 20.4 & 3.1 & - \\
\hline Acetonitrile & 21.5 & 73.3 & 22.8 & 3.9 & - \\
\hline Methanol & 14.3 & 39.0 & 26.1 & 6.3 & $28.6^{\mathrm{a}}$ \\
\hline
\end{tabular}

Reaction conditions: catalyst $=100 \mathrm{mg}$, substrate:TBHP: solvent $=1: 2: 5$ (mole ratio), $T=333 \mathrm{~K}, t=6 \mathrm{~h}$.

${ }^{a}$ Methyl benzoate.

Table 2

Catalytic activity of V-MCM-48 for toluene oxidation with $70 \%$ TBHP

\begin{tabular}{|c|c|c|c|c|c|}
\hline \multirow[t]{2}{*}{ Solvent } & \multirow[t]{2}{*}{ Conversion (\%) } & \multicolumn{4}{|c|}{ Product selectivity (\%) } \\
\hline & & Benzaldehyde & Benzoic acid & Benzyl alcohol & Others \\
\hline None & 5.5 & 71.4 & 20.0 & 5.1 & 3.5 \\
\hline Acetone & 23.2 & 74.4 & 21.1 & 4.5 & - \\
\hline Acetonitrile & 19.4 & 70.9 & 23.9 & 2.5 & 3.0 \\
\hline Methanol & 11.2 & 34.4 & 24.0 & 10.5 & $31.1^{\mathrm{a}}$ \\
\hline
\end{tabular}

Reaction conditions: catalyst $=100 \mathrm{mg}$, substrate:TBHP: solvent $=1: 2: 5$ (mole ratio), $T=333 \mathrm{~K}, t=6 \mathrm{~h}$.

${ }^{a}$ Methyl benzoate. 
Table 3

Comparative activity of various catalysts for toluene oxidation with $30 \% \mathrm{H}_{2} \mathrm{O}_{2}$

\begin{tabular}{|c|c|c|c|c|c|c|c|}
\hline \multirow[t]{2}{*}{ Catalyst } & \multirow[t]{2}{*}{ Conversion $(\%)$} & \multicolumn{6}{|c|}{ Product selectivity (\%) } \\
\hline & & Benzaldehyde & Benzoic acid & Benzyl alcohol & O-cresol & P-cresol & Others \\
\hline V-ALPO & 28.4 & 64.4 & 4.1 & 4.0 & 12.0 & 9.8 & 1.0 \\
\hline V-MCM-48 & 22.7 & 67.2 & 6.0 & 1.5 & 15.5 & 10.5 & 4.0 \\
\hline V-MCM-41 [20] & 20.8 & 62.0 & - & 2.0 & 20.0 & 14.0 & 2.0 \\
\hline V-Al-Beta [21] & 14.0 & 56.0 & - & 4.0 & 21.0 & 17.0 & 2.0 \\
\hline VS-1 [22] & 11.7 & 52.2 & - & 7.7 & 19.7 & 17.1 & 3.7 \\
\hline
\end{tabular}

Reaction conditions: catalyst $=100 \mathrm{mg}$, toluene: $30 \% \mathrm{H}_{2} \mathrm{O}_{2}$ :acetonitrile $=3: 1: 10, T=353 \mathrm{~K}, t=24 \mathrm{~h}$.

was continued with the filtrate. No increase in conversion was observed, thus indicating that homogeneous catalysis is not taking place under the reaction conditions.

For comparison, the same reaction has been performed with $30 \% \mathrm{H}_{2} \mathrm{O}_{2}$ with acetonitrile as solvent and results are given in Table 3. V-AlPO is more active and selective to benzaldehyde over other V-substituted molecular sieves. A considerable quantity of cresols have been observed in all the cases. It was observed that V-MCM-48 shows a slightly higher conversion compared to V-MCM-41. This could be due to the interwoven network of cubic MCM-48 where the diffusional constraints are less compared to the onedimensional hexagonal MCM-41 network. The higher activity of V-substituted mesoporous materials suggests high dispersion of vanadium in mesoporous framework, which is a prerequisite for the oxidation reactions.

\section{Conclusion}

The incorporation of vanadium in the framework of aluminium phosphate materials has been achieved through structure directing template route. Vanadium $\left(\mathrm{V}^{4+} / \mathrm{V}^{5+}\right)$ substituted AlPOs have been shown to be promising oxidising catalysts with $70 \%$ TBHP and $30 \% \mathrm{H}_{2} \mathrm{O}_{2}$ as oxidising agents. In fact, toluene has been oxidized into benzaldehyde with a close to $80 \%$ selectivity at a conversion rate close to $30 \%$.

V-AlPO showed higher activity as compared with that of other similar porous materials like V-MCM48 and V-MCM 41.

\section{Acknowledgements}

The authors are grateful to Dr. Kiwi-Minsker for fruitful discussions and to Mr. N. Xanthopoulos and P. Möckli for kindly providing us the XPS and XRD data reported in this paper. We are also thankful to RSIC, IIT-Bombay for ICP analysis.

\section{References}

[1] S.T. Wilson, B.M. Lok, C.A. Messina, T.R. Cannan, E.M. Flanigen, J. Am. Chem. Soc. 104 (1982) 1176.

[2] E.M. Flanigen, B.M. Lok, R.L. Patton, S.T. S Wilson, in: Proceedings of the Seventh International Zeolite Conference, Tokyo, Japan, 1986, p. 103.

[3] T. Kresge, M.E. Leonowicz, W.J. Roth, J.C. Vartuli, J.S. Beck, Nature 359 (1992) 710.

[4] T. Kimura, Y. Sugahara, K. Kuroda, Phosph. Res. Bull. 6 (1996) 205.

[5] T. Kimura, Y. Sugahara, K. Kuroda, Chem. Commun. (1998) 559.

[6] T. Kimura, Y. Sugahara, K. Kuroda, Chem. Mater. 11 (1999) 508.

[7] Feng, Y. Xia, J. Feng, X. Bu, G.D. Stucky, Chem. Commun. (1997) 949.

[8] S. Ayyappan, C.N.R. Rao, Chem. Commun. (1997) 575.

[9] B. Chakraborty, A.C. Pulikottil, S. Das, B. Viswanathan, Chem. Commun. (1997) 911.

[10] D. Zhao, Z. Luan, L. Keven, Chem. Commun. (1997) 1009.

[11] M.P. Kapoor, A. Raj, Appl. Catal. A 203 (2000) 311.

[12] X.S. Zhao, G.Q. Lu, Micropor. Mesopor. Mater. 44-45 (2001) 185.

[13] D. Zhao, Z. Luan, L. Kevan, J. Phys. Chem. 101 (1997) 6943.

[14] Ch. Subrahmanyam, B. Louis, F. Rainone, B. Viswanathan, A. Renken, T.K. Varadarajan, Catal. Commun. 3 (2002) 45.

[15] Ch. Subrahmanyam, B. Louis, F. Rainone, B. Viswanathan, A. Renken, T.K. Varadarajan, Appl. Catal. A 3 (2002) 45.

[16] Ch. Subrahmanyam, B. Viswanathan, T.K. Varadarajan, J. Mol. Catal. A 223 (2004) 149.

[17] S.K. Mahapatra, B. Sahoo, W. Kenue, P. Selvem, Chem. Commun. (2002) 1466.

[18] J.C. Vartuli, K.D. Schmitt, C.T. Kresge, W.J. Roth, M.E. Leonowicz, S.B. McCullen, S.D. Hellring, J.S. Beck, J.L. Schlenker, D.H. Olson, E.W. Sheppard, Chem. Mater. 6 (1994) 2317.

[19] T. Sen, P.R. Rajamohanan, S. Ganapathy, S. Sivasankar, J. Catal. 163 (1996) 354

[20] M. Chatterjee, T. Iwasaki, H. Hayashi, Y. Onodera, T. Ebina, T. Nagase, Chem. Mater. 11 (1999) 1368.

[21] T. Sen, M. Chatterjee, S. Sivasanker, Chem. Commun. (1995) 207.

[22] P.H. Hari Prasad Rao, A.A. Belhekar, S.G. Hegde, A.V. Ramaswamy, P. Ratnasamy, J. Catal. 141 (1993) 595. 